\title{
Relation of UTI with Type 2 Diabetes and Pregnancy in Women Attended Obstetrics, Gynecology and Pediatric Hospital in Kirkuk City
}

\author{
Jaklen Zaea khoshaba ${ }^{1}$, Shaimaa Mohammed Sulaiman ${ }^{1}$, \\ Sundus Abdulkaream Mardan', Muhannad Abdullah Alazzawy ${ }^{2}$ \\ ${ }^{I}$ M.B.Ch. B.D.G.O., ${ }^{2}$ Ph.D., Medical Microbiology, Kirkuk Health Directorate, Kirkuk, Iraq
}

\begin{abstract}
The study was conducted in the city of Kirkuk in the period from October 2019 to March 2020, which included 100 pregnant women with type 2 diabetes who attended obstetric, gynaecology and paediatric hospital in Kirkuk city and 100 people intact and without any chronic disease .. The study included the laboratory examination of the reactions samples, where close quantities of midstream urine were collected for both groups. After collecting the calories, the calories were examined microscopically, and then the reactions were transplanted into the available culture media to isolate the aetiology of urinary tract infections such as blood agar and MacConkey agar, and the CLED agar to isolate and diagnose bacteria of all kinds .. In addition, the sensitivity test for antibiotics was applied by the feeding medium where the method were relied upon Global standard bacteriological culture procedure, isolation and diagnosis by biochemical tests. Among diabetes mellitus patients included in this study, $80 \%$ were positive for UTI patients, while $20 \%$ were negative for UTI. According to the distribution of the isolated bacteria among the study groups, the common isolated bacteria among DM was $E$.coli which constituted $26.36 \%$ followed by $K$. pneumoniae which constituted $17.27 \%$, S. aureus and P. mirabilis which constituted $8.18 \%$ and the lowest percentage was Enterobacter cloacae for $1.81 \%$. In this study $S$. aureus showed high rate of sensitivity to ciprofloxacin and oxacillin $(88.23 \%)$ while it was resistant to ampicillin and lincomycin with rate of $100 \%$. Staphylococcus epidermidis showed high rate of sensitivity to tobramycin and oxacillin $(92.85 \%)$ while it was resistant to ampicillin, lincomycin and amoxicillin (100\%) Escherichia coli showed high rate of sensitivity (93.02\%) to ceftazidim and it was $100 \%$ resistant to oxacillin and lincomycin. Klebsiella pneumoniae showed high rate of sensitivity $(81.48 \%)$ to amoxiclave and it was resistant to ampicillin, tetracycline, erythromycin, oxacillin and lincomycin (100\%).
\end{abstract}

Keywords: UTI; Type 2 Diabetes; Pregnancy.

\section{Introduction}

Urinary tract infections (UTIs) are frequently encountered in pregnant women. Pyelonephritis is the most common serious medical condition seen in pregnancy. Thus, it is crucial for providers of obstetric care to be knowledgeable about normal findings of the urinary tract, evaluation of abnormalities, and treatment of disease ${ }^{(1)}$. During pregnancy, urinary tract changes predispose women to infection. Ureteral dilation is seen due to compression of the ureters from the gravid uterus. Hormonal effects of progesterone also may cause smooth muscle relaxation leading to dilation and urinary stasis, and vesicoureteral reflux increases ${ }^{(2)}$.
Bacterial contamination of the urine within the urinary tract (bacteriuria) is common and can at times result in microbial invasion of tissue responsible for the formation, transport and storage of urine, Pathogenrelated conditions (such as the presence of invasion or virulence factors) affect the severity of the infection and its resistance to antibiotic therapy, but also different host related characteristics have been individuated, that play a role in particular in the possibility of infection recurrence $^{(3)}$. Urinary tract infection may involve only the lower urinary tract or both the upper and the lower tracts $^{(1)}$. The term cystitis has been used to describe the syndrome involving dysuria, frequency, 
and occasionally suprapubic tenderness ${ }^{(4)}$. More than $95 \%$ of urinary tract infections are caused by a single bacterial species. Escherichia coli is the most frequent infecting organism in acute infection ${ }^{(4)}$. Other organisms that can be responsible for UTIs include Gram positive cocci, such as Enterococcus faecalis, Staphylococcus aureus and coagulase negative staphylococci (CoNS). Other Gram negative organisms responsible for causing UTIs include Klebsiella species, Proteus species, Pseudomonas aeroginosae and Enterobacter species $^{(5)}$. The aim of the study was to evaluate the relation of UTI with type 2 diabetes

\section{Material and Method}

The study was conducted in the city of Kirkuk in the period from October 2019 to March 2020, which included 100 pregnant women with type 2 diabetes who attended obstetric, gynaecology and paediatric hospital in Kirkuk city and without any chronic disease .. The study included the laboratory examination of the reactions samples, where close quantities of median reactions were collected for both groups. After collecting the calories, the calories were examined microscopically, and then the reactions were transplanted into the available culture media to isolate the etiology of urinary tract infections such as blood agar and MacConkey agar, and the CLED agar to isolate and diagnose bacteria of all kinds .. In addition, the sensitivity test for antibiotics was applied by the feeding medium where the method were relied upon Global standard bacteriological culture procedure, isolation and diagnosis by biochemical tests. Type 2 diabetes has been defined as any person with diabetes who is over 15 years old and who uses oral treatment, not insulin.

Finding: Among diabetes mellitus patients included in this study, $80 \%$ were positive for UTI patients, while $20 \%$ were negative for UTI. Patients without DM showed no positivity for UTI ... Table 1 .

\section{Table 1: Relation of UTI with diabetes}

\begin{tabular}{|l|c|c|c|c|}
\hline \multirow{2}{*}{ Characteristics } & \multicolumn{2}{|c|}{ Diabetic } & \multicolumn{2}{c|}{ Control group } \\
\cline { 2 - 5 } & No. & $\mathbf{\%}$ & No. & \% \\
\hline With UTI & 80 & 80 & 0 & 0 \\
\hline Without UTI & 20 & 20 & 100 & 100 \\
\hline Total & $\mathbf{1 0 0}$ & $\mathbf{1 0 0}$ & $\mathbf{1 0 0}$ & $\mathbf{1 0 0}$ \\
\hline
\end{tabular}

P. value 0.003

The study showed that majority of patients with UTI were suffered from G+ve bacteria as compared with DM patient without UTI.

Table 2: Results of urine culture among study groups.

\begin{tabular}{|l|c|c|c|c|}
\hline \multirow{2}{*}{ Results of urine culture } & \multicolumn{3}{|c|}{ DM patients } \\
\cline { 2 - 5 } & \multicolumn{2}{|c|}{ With UTI } & \multicolumn{3}{|c|}{ Without UTI } \\
\cline { 2 - 5 } & No. & No. & 5 & 25 \\
\hline Positive Bacterial culture & 50 & 62.5 & 15 & 75 \\
\hline Negative Bacterial Culture & 30 & 37.5 & $\mathbf{2 0}$ & $\mathbf{1 0 0}$ \\
\hline Total & $\mathbf{8 0}$ & $\mathbf{1 0 0}$ & \multicolumn{3}{|c|}{} \\
\hline
\end{tabular}

P. value 0.002

In the present study the ages of the patients ranged between 16 and 65 years old. As shown in Table 3, the highest percentage of the patients among DM women were within the age group 26-35 years old constituted $44 \%$. The lowest percentage was within the age group of 16-25 years old which constituted $31.03 \%$. 
Table 3: Distribution of positive urine culture among pregnant and non-pregnant women according to age groups.

\begin{tabular}{|c|c|}
\hline Age group (years) & $\mathbf{\%}$ \\
\hline $16-25$ & 31.03 \\
\hline $26-35$ & 44 \\
\hline $36-45$ & 42.10 \\
\hline $46-55$ & 34.69 \\
\hline $56-65$ & 41.17 \\
\hline
\end{tabular}

Table 4: Distribution of isolated bacteria among studied patients.

\begin{tabular}{|l|c|c|}
\hline \multirow{2}{*}{ Isolated Bacteria } & \multicolumn{2}{|c|}{ DM } \\
\cline { 2 - 3 } & No & \% \\
\hline Escherichia coli & 29 & 26.36 \\
\hline Staphylococcus aureus & 9 & 8.18 \\
\hline Streptococcus faecalis & 8 & 7.27 \\
\hline Serratia murcescens & 5 & 4.54 \\
\hline Klebsiella pneumoniae & 19 & 17.27 \\
\hline Enterobacter cloacae & 2 & 1.81 \\
\hline Proteus mirabilis & 9 & 8.18 \\
\hline Proteus vulgaris & 6 & 5.45 \\
\hline Staphylococcus epidermidis & 7 & 6.36 \\
\hline Staphylococcus saprophyticus & 8 & 7.27 \\
\hline Pseudomonas aeruginosa & 8 & 7.27 \\
\hline Total & $\mathbf{1 1 0}$ & $\mathbf{1 0 0}$ \\
\hline
\end{tabular}

P. value 0.048

According to the distribution of the isolated bacteria among the study groups, as shown in Table 4, the common isolated bacteria among DM was $E$.coli which constituted $26.36 \%$ followed by $K$. pneumoniae which constituted $17.27 \%, S$. aureus and $P$. mirabilis which constituted $8.18 \%$ and the lowest percentage was Enterobacter cloacae for $1.81 \%$.

In this study $S$. aureus showed high rate of sensitivity to ciprofloxacin and oxacillin (88.23\%) while it was resistant to ampicillin and lincomycin with rate of $100 \%$. Staphylococcus epidermidis showed high rate of sensitivity to tobramycin and oxacillin $(92.85 \%)$ while it was resistant to ampicillin, lincomycin and amoxicillin (100\%). Staphylococcus saprophyticus showed high rate of sensitivity to cephalothin, ciprofloxacin, oxacillin and lincomycin while it was $100 \%$ resistant to ampicillin, erythromycin and amikacin. Streptococcus faecalis showed $100 \%$ sensitivity to oxacillin while it was resistant to ciprofloxacin, ampicillin, erythromycin and amikacin. Escherichia coli showed high rate of sensitivity (93.02\%) to ceftazidim and it was $100 \%$ resistant to oxacillin and lincomycin. Klebsiella pneumoniae showed high rate of sensitivity $(81.48 \%)$ to amoxiclave and it was resistant to ampicillin, tetracycline, erythromycin, oxacillin and lincomycin (100\%). Proteus mirabilis showed high sensitivity rate to cefotaxim (94.44\%) and it was resistant to erythromycin, ampicillin, oxacillin and lincomycin (100\%). Proteus vulgaris showed high sensitivity rate to nirtrofurontoin, cefotaxim $(92.85 \%)$ and low rate of sensitivity to erythromycin. Pseudomonas aeroginosae showed high sensitivity rate to cefotaxim $(86.66 \%)$ and low rate of sensitivity (20\%) to ampicillin. Enterobacter cloacae showed high sensitivity rate to cefotaxim $(100 \%)$ and it was resistant to erythromycin, tobramycin, oxacillin, streptomycin, cephalothin and amikacin $(100 \%)$.All these results were summarized in Table 5.

Table 5: Distribution of Antibiotics Sensitivity of Gram Positive Bacteria Isolated from Married Women with UTI.

\begin{tabular}{|l|c|c|c|c|c|c|c|}
\hline Type of antibiotic & S. aureus & $\begin{array}{c}\text { S. } \\
\text { epidermidis }\end{array}$ & $\begin{array}{c}\text { S.s } \\
\text { saprophyticus }\end{array}$ & E. coli & $\begin{array}{c}\text { K. } \\
\text { pneumoniae }\end{array}$ & P. mirabilis & $\begin{array}{c}\text { P. } \\
\text { aeruginosa }\end{array}$ \\
\hline Ciprofloxacin & 88.23 & 50 & 56.25 & 79.06 & 59.25 & 77.77 & 73.33 \\
\hline Ampicillin & 0 & 0 & 0 & 6.97 & 0 & 0 & 20 \\
\hline Tetracycline & 47.05 & 28.57 & 43.75 & 30.23 & 0 & 11.11 & 53.33 \\
\hline Erythromycin & 70.58 & 50 & 0 & 6.97 & 0 & 0 & 26.66 \\
\hline Tobramycin & 82.35 & 92.85 & 50 & 65.11 & 22.22 & 72.22 & 53.33 \\
\hline Gentamicin & 82.35 & 85.71 & 50 & 32.55 & 40.74 & 77.77 & 60 \\
\hline Oxacillin & 88.23 & 92.85 & 56.25 & 0 & 0 & 0 & 26.66 \\
\hline
\end{tabular}




\begin{tabular}{|l|c|c|c|c|c|c|c|}
\hline Penicillin G & 5.88 & 0 & 12.5 & 30.23 & 25.92 & 66.66 & 73.33 \\
\hline Streptomycin & 70.58 & 57.14 & 50 & 65.11 & 59.25 & 44.44 & 80 \\
\hline Chloramphenicol & 58.82 & 50 & 6.25 & 18.60 & 48.14 & 27.77 & 26.66 \\
\hline Trimethoprim & 70.58 & 50 & 50 & 4.65 & 22.22 & 16.66 & 40 \\
\hline Clindamycin & 58.82 & 57.14 & 50 & 0 & 0 & 0 & 26.66 \\
\hline Lincomycin & 0 & 0 & 56.25 & 93.02 & 33.33 & 72.22 & 53.33 \\
\hline Ceftazidim & 58.82 & 50 & 43.75 & 11.62 & 22.22 & 27.22 & 46.66 \\
\hline Amoxicillin & 11.76 & 0 & 37.5 & 23.25 & 14.81 & 44.44 & 33.33 \\
\hline Cephalothin & 58.82 & 85.71 & 75 & 76.74 & 22.22 & 83.33 & 66.66 \\
\hline Amikacin & 47.05 & 71.42 & 0 & 81.39 & 81.48 & 88.88 & 53.33 \\
\hline Amoxiclave & 47.05 & 50 & 50 & 81.39 & 70.37 & 77.77 & 73.33 \\
\hline Nitrofurantoin & 47.05 & 64.28 & 50 & 86.04 & 77.77 & 94.44 & 86.66 \\
\hline Cefotaxime & 41.17 & 71.42 & 50 & 79.06 & 59.25 & 77.77 & 73.33 \\
\hline
\end{tabular}

\section{Discussion}

Urinary tract infection(UTI) in pregnant women with DM have special importance due to complication that results from its which can be dangerous to both mother and baby ${ }^{(6)}$. Diabetes mellitus has long been considered to be a predisposing factor for urinary tract infection ${ }^{(7)}$. Among DM patients included in this study, 82.14\% were positive for UTI among married women. This clarifying the significance of the relation between DM and the occurrence of UTI among married women in this study which supported by other study, which showed that urinary tract infection is more prevalent among married women with $\mathrm{DM}{ }^{(8,9)}$. Nasir in his study found that there is an increase in the prevalence of asymptomatic pyuria among diabetic females with complications of retinopathy and nephropathy ${ }^{(10)}$. The choice of antibiotic depends on the spectrum and susceptibility patterns of the uropathogens, its effectiveness for this indication, its collateral effects and coast ${ }^{(11)}$. In current study Staphylococcus aureus showed high sensitivity to ciprofloxacin and oxacillin $88.23 \%$ and it was resistant to ampicillin and lincomycin with rate of $100 \%$. Staphylococcus epidermidis showed high sensitivity to tobramycin and oxacillin with rate of $92.85 \%$, while it was $100 \%$ resistant to each of ampicillin, lincomycin and amoxicillin. Staphylococcus saprophyticus was $100 \%$ resistant to each of ampicillin, erythromycin and amikacin. Streptococcus faecalis showed 100\% sensitivity to oxacillin while it was resistant in high rate to ciprofloxacin, ampicillin, erythromycin and amikacin. A study done by Amin et al ${ }^{(12)}$ showed that the most effective antibiotics against Gram -positive cocci were kanamycin, tobramycin and ciprofloxacin. A study by Mahdi (13) who reported that gentamicin is the most effective antibiotic against Staphylococcus aureus. . The current study was in agreement with $\operatorname{Kandela}^{(167)}$ who reported that multi resistance to antibiotics ranging between (7-19) antibiotics, and all isolates of $K$. pneumoniae were resistant $100 \%$ to ampicillin, cephalexin, amoxicillin, penicillin, tetracycline, gentamycin and amikacin, in addition the isolates showed high resistance to third generation of cephalosporin included $60 \%$ resistant to ceftazidim and $80 \%$ resistant to cefotaxim and ceftriaxone. Imipenem and azteronem was found to be the most effective agents against the isolates. Amin ${ }^{(12)}$ in his study found that Gram negative bacilli were responsible for UTI infections, the most common isolated bacteria from urinary infections were $E$. coli and the most effective antimicrobial agents were amikacin, tobramycin and ciprofloxacin against Gram negative bacilli. A study by Fakhriddeen ${ }^{(15)}$ reported that the result of sensitivity appeared that most of used antibiotics were ineffective on $E$. coli isolates with exception of amikacin meropenem and ciprofloxacin respectively. A study done by $\mathrm{Wu}$ et $a l^{(16)}$ reported that most bacterial isolates exhibited sensitivity to antibiotics norofloxacin, cefotaxim, were the more effective antibiotics on bacterial isolates, while gentamicin, streptomycin and ultracloxam showed low effect. The highly sensitivity of all bacterial isolates to norofloxacin lead to consider that this drug is the best for treatment of UTI. 


\section{Conclusion}

It was concluded that there was a significant relation of UTI with T2D and E. coli plus S. aureus were the predominate causes

Financial Disclosure: There is no financial disclosure.

Conflict of Interest: None to declare.

Ethical Clearance: All experimental protocols were approved under the Kirkuk Health Directorate and all experiments were carried out in accordance with approved guidelines.

\section{References}

1. Daniele M, Gianluca D, Alessandro C, Giovanni M. Urinary tract infections and dysfunctional voiding. in: Peter Tenke. Urinary tract infection. Rijeka : InTech; 2011:6-50.

2. Nicolle LE, Bradley S, Colgan R, Rice JC, Schaeffer A, Hooton TM. Infectious Diseases Society of America Guidelines for the diagnosis and treatment of asymptomatic bacteriuria in adults. Clin Infect Dis 2005;40:643-654.

3. Foxman B, Geiger A M, Palin K, Uespie G, Koopman J. First-time urinary tract infection and Sexual Behavior. Epidemiology 1995; 6 : 162-168.

4. Makinkandan S G, Singh M, Kumaraguru A. Emerging of multidrug resistance human pathogen from urinary tract infection. Current Res Bacteriol 2011;4:9-15.

5. Baraboutis, Tsagalou, Lepinski, et al. Primary Staphylococcus aureus urinary tract infection:the role of undetected hematogenous seeding of the urinary tract. Eur J Clinical Microbiol Infect 2011;12:1095-1101.

6. Dakeel K H, Taktuk N K . Isolation and diagnosis of bacteria causing urinary tract infection in pregnant women with diabetes mellitus type 2 and its resistance to antibiotics .Eng and Technol J 2009; 27(16) :577-594.

7. Al-Falahi A H, Al-Falahi R H .Bacterial etiological agents associated with urinary tract infection and their antibiotic susceptibility in diabetic and nondiabetic women. Al-Taqni 2010;23(3):43-48.

8. Al-Bayaa Y J. Genetourinary tract infection in diabetic women :Bacteriological study. J Fac Med Baghdad 2010; 52(3) :320-323.

9. Conforth T. Womens health . Urinary Tract Infect 2010; 1(3):5-7

10. Nasir H A. Asymptomatic pyuria in diabetic females. J Fac Med Baghdad 2006;48 (3) 274-276.

11. Naber KG, Wullt B, Wagenlehner F M. Antibiotic treatment of uncomplicated urinary tract infection premenopausal women. Inter $\mathrm{J}$ antimicrob agents 2011; 285:21-35.

12. Amin M, Mehdinejad M, Pourdangchi Z. Study of bacteria isolated from urinary tract infections and determination of their susceptibility to antibiotics. Jundishapur J Microbiol 2009;2(3):118-123.

13. Mahdi A Gh. Isolation \& Identification of aerobic bacteria Causing Urinary Tract Infection in Pregnant Women in Al-Diwaniya city and its sensitivity to some antibiotics. J Al-Qadisiyah pure Sc. $2009 ; 14(1): 1-12$

14. Kandela N J. Detection of extended spectrum beta -lactamase (ESBL) and klobocin production from Klebsiella pneumoniae local isolates from urinary tract infections. AJPS 2011;11(1):21-42.

15. Fakhridden A J . Occurrence of Escherichia coli in patients with urinary tract infections in Najaf city. Kufa J Vet Med Sc 2011; 2(2) :19-29.

16. Wu H J, Wang A, Jennings M. Discovery of virulence factors of pathogenic bacteria .Curr Openion Chem Biol 2008; 12: 1-9. 